\title{
INVARIANTS AND ASYMPTOTIC BEHAVIOR OF SOLUTIONS OF A CONSERVATION LAW
}

\author{
TAI-PING LIU ${ }^{1}$
}

\begin{abstract}
We study the asymptotic behavior of solutions of the initial value problem for a conservation law $u_{t}+f(u)_{x}=0$. It is assumed that the initial data agrees with the Riemann data for $|x|$ large. We show that the solution approaches that of the corresponding Riemann problem at algebraic rates.
\end{abstract}

We consider the initial value problem for single conservation laws

$$
\begin{gathered}
u_{t}+f(u)_{x}=0, \quad t \geqslant 0,-\infty<x<\infty, \\
u(x, 0)=u_{0}(x), \quad-\infty<x<\infty,
\end{gathered}
$$

where $f$ is a smooth function of $u, u=u(x, t)$. In this paper we will consider piecewise continuous weak solutions of (1). Across a discontinuity $x=x(t)$, the solution satisfies the Rankine-Hugoniot condition $(\mathrm{R}-\mathrm{H})$ and the entropy condition (E), [8],

(R-H) $x^{\prime}(t)=\sigma\left(u_{-}, u_{+}\right)$,

(E) $\sigma\left(u_{-}, u_{+}\right) \leqslant \sigma\left(u_{-}, u\right)$ for all $u$ between $u_{-}$and $u_{+}$, where $u=u( \pm x(t), t)$ and $\sigma\left(u_{1}, u_{2}\right)$ is the shock speed defined as

$$
\sigma\left(u_{1}, u_{2}\right)=\frac{f\left(u_{1}\right)-f\left(u_{2}\right)}{u_{1}-u_{2}} .
$$

An easy consequence of condition $(E)$ is the following shock inequalities

$$
\lambda_{+} \equiv f^{\prime}\left(u_{+}\right) \leqslant \sigma\left(u_{-}, u_{+}\right) \leqslant f^{\prime}\left(u_{-}\right) \equiv \lambda_{-} .
$$

We will investigate the solution $u(x, t)$ of (1), (2) when the initial data satisfy

$$
u_{0}(x)= \begin{cases}u_{l} & \text { for } x \leqslant-S / 2 \\ u_{r} & \text { for } x \geqslant S / 2\end{cases}
$$

for some constants $u_{l}, u_{r}$ and $S, S>0$. For the asymptotic behavior and invariants of solutions when $u_{l}=u_{r}$, see Dafermos [1], DiPerna [2], Keyfitz [5] and Lax [6], [7]. Our results overlap with some of the recent results of Dafermos [9].

When $u_{r} \geqslant u_{l}$, we set

Received by the editors August 8, 1977 and, in revised form, February 21, 1978.

AMS (MOS) subject classifications (1970). Primary 35L65; Secondary 35F25.

Key words and phrases. Conservation laws, entropy condition, shock inequalities, $L_{1}$-invariants.

${ }^{1}$ Supported by NSF Grant MCS 75-05267. 
$p(t)=\min _{x} \int_{-\infty}^{x}\left(u(y, t)-u_{l}\right) d y, \quad q(t)=\max _{x} \int_{x}^{+\infty}\left(u(y, t)-u_{r}\right) d y$.

THEOREM 1. (i) $p^{\prime}(t) \geqslant 0$ and $q^{\prime}(t) \leqslant 0$,

(ii) $p^{\prime}(t)=p=$ constant and $q(t)=q=$ constant if $f^{\prime \prime}>0$.

Proof. The following function is defined independently of the path of integration because of (1):

$$
\varphi(x, t)=\int_{(-\infty, 0)}^{(x, t)}\left(u(x, t)-u_{l}\right) d x-\left(f(u(x, t))-f\left(u_{l}\right)\right) d t .
$$

It follows easily that $\varphi$ satisfies

$$
\varphi_{t}+\frac{f(u)-f\left(u_{l}\right)}{u-u_{l}} \varphi_{x}=0
$$

From (5) and (6), we have $p(t)=\min _{x} \varphi(x, t)$. Suppose that the minimum at time $t=t_{0}$ takes place at $x=x_{0}$. We draw a curve $\chi$ through $\left(x_{0}, t_{0}\right)$ with

$$
\frac{d x}{d t}=\frac{f(u(x, t))-f\left(u_{l}\right)}{u(x, t)-u_{l}}
$$

We note that $\varphi=$ constant along $\chi$ according to (7). Thus to prove (i) we need only to show that $\chi$ is defined for $t \in\left(t_{0}-\varepsilon, t_{0}\right)$ for some $\varepsilon>0$. Similarly to prove (ii) we have to show that when $f^{\prime \prime}>0, \chi$ is defined for $t \in\left(t_{0}-\varepsilon, t_{0}+\varepsilon\right)$ for some $\varepsilon>0$. Thus we need only treat the case where $u$ is not continuous at $\left(x_{0}, t_{0}\right)$. When $f^{\prime \prime}>0$, this cannot be the case because $u$ decreases from left to right across a discontinuity by virtue of (3). This proves (ii) for $p(t)$. In the general case, if $\chi$ is not defined for $t \in\left\{t_{0}-\varepsilon, t_{0}\right\}$, then

$$
\frac{f\left(u_{-}\right)-f\left(u_{l}\right)}{u_{-}-u_{l}}<\sigma\left(u_{-}, u_{+}\right)<\frac{f\left(u_{+}\right)-f\left(u_{l}\right)}{u_{+}-u_{l}}
$$

where $u_{ \pm}=u\left(x_{0} \pm 0, t\right)$. It is easy to see that (9) contradicts the entropy condition (E). This proves (i) for $p(t)$. The proof for $q(t)$ is analogous. Q.E.D.

In what follows we always assume that $f^{\prime \prime} \neq 0$. For definiteness, we assume that

$$
f^{\prime \prime}(u)>0 \text { for all } u .
$$

It follows from (1) that $u$ is constant along the characteristic curve $d x / d t=$ $\lambda(x, t), \lambda=f^{\prime}(u)$. Thus characteristic curves are straight lines. A generalized characteristic curve, [3], is a Lipschitz continuous curve with characteristic on shock speeds. Through $(0,-S / 2)$ and $(0, S / 2)$ we draw generalized characteristics $\chi_{1}: x=x_{l}(t)$ and $\chi_{2}: x=x_{r}(t)$, respectively. We note that when $f^{\prime \prime}(u) \neq 0$, the solution does not contain centered rarefaction wave except perhaps when $t=0$. Thus $\chi_{1}$ and $\chi_{2}$ are well defined and $u(x, t)=u_{l}$ $\left(u_{r}\right)$ when $x>x_{l}(t)\left(<x_{r}(t)\right)$. 
THEOREM 2. If $u_{r}<u_{l}$, then there exists $t_{0}, t_{0} \geqslant 0$, such that for $t \geqslant t_{0}$, the solution $u(x, t)$ of $(1),(2)$ is of the form:

$$
u(x, t)= \begin{cases}u_{l} & \text { for } x-\sigma t \leqslant x_{0} \\ u_{r} & \text { for } x-\sigma t \geqslant x_{0}\end{cases}
$$

where $\sigma=\sigma\left(u_{l}, u_{r}\right)$ and

$$
x_{0}=\frac{1}{u_{r}-u_{l}} \int_{-N}^{N}\left(\frac{u_{r}+u_{l}}{2}-u_{0}(x)\right) d x, \quad N \geqslant \frac{S}{2} .
$$

Proof. Let us set $u^{+}(t)=u\left(x_{l}(t)+0, t\right), u^{-}(t)=u\left(x_{r}(t)-0, t\right)$ and $\lambda^{ \pm}(t)=f^{\prime}\left(u^{ \pm}(t)\right)$. Since $\chi_{1}$ and $\chi_{2}$ propagate with shock or characteristic speeds, we have

$$
D^{\prime}(t)=x_{r}^{\prime}(t)-x_{l}^{\prime}(t)=\sigma\left(u^{-}, u_{r}\right)-\sigma\left(u_{l}, u^{+}\right)
$$

which, according to (E) and (14), lies between $\lambda^{-}-\lambda^{+}$and $\lambda_{r}-\lambda_{l}$. Thus it follows from the mean value theorem that for some $\theta \in(0,1)$,

$$
D^{\prime}(t) \leqslant \theta\left(\lambda^{-}-\lambda^{+}\right)+(1-\theta)\left(\lambda_{r}-\lambda_{l}\right) .
$$

Through $\left(x_{l}(t)+0, t\right)$ and $\left(x_{r}(t)-0, t\right)$ we draw characteristics backward in time. These characteristics do not meet shock waves because of (3). Thus they are straight lines with speeds $\lambda^{ \pm}$and meet $t=0$ between $\chi_{1}$ and $\chi_{2}$. Thus we have

$$
D(t) \leqslant\left[\lambda^{-}(t)-\lambda^{+}(t)\right] t
$$

The above estimate and (12) imply that

$$
D(t) \leqslant\left\{D(1)-\left(\lambda_{r}-\lambda_{l}\right) t^{\theta}+\left(\lambda_{r}-\lambda_{l}\right) t .\right.
$$

Thus $D(t)=0$ for $t$ large. It remains to find $x_{0}$. Take $K$ sufficiently large so that $u(x, t)=u_{r}$ for $x-\sigma t=K$. It is easy to see from (1) and (R-H) that

$$
\eta(t)=\int_{-\infty}^{K+\sigma t}\left(u-u_{l}\right) d x
$$

is time invariant. For $t \geqslant t_{0}, \eta(t)=\left(u_{r}-u_{l}\right)\left(K-x_{0}\right)$. Equating this with $\eta(0)$ we obtain the value of $x_{0}$. Q.E.D.

A generalized $N$-wave is a rarefaction bracketed by two shock waves:

$$
N\left(x, t ; p, q ; u_{l}, u_{r}\right)= \begin{cases}u_{l} & \text { for } x-\lambda_{l} t \leqslant-\sqrt{-2 p f^{\prime \prime}\left(u_{l}\right) t} \\ u_{r} & \text { for } x-\lambda_{r} t \geqslant \sqrt{2 q f^{\prime \prime}\left(u_{r}\right) t} \\ u(\xi) & \text { otherwise }\end{cases}
$$

where $u(\xi)$ is the unique value of $u$ with

$$
\lambda(\xi)=f^{\prime}(u(\xi))=\xi, \quad \xi=x / t
$$

THEOREM 4. Suppose that $u(x, t)$ is the solution of (1), (2) and $u_{r} \geqslant u_{l}$. Then for $p, q$ of (6) and $N(x, t)=N\left(x ; t, p, q ; u_{l}, u_{r}\right)$ of $(15), \Lambda \equiv \max _{(x, t)}$ $f^{\prime \prime}(u(x, t))$, we have 
(i) The edges of $N$ and $u$ have finite distance for all $t$, i.e.

$$
\left|x_{l}(t)-\left(\lambda_{l} t-\sqrt{-2 p f^{\prime \prime}\left(u_{l}\right) t}\right)\right|+\left|x_{r}(t)-\left(\lambda_{r} t+\sqrt{2 q f^{\prime \prime}\left(u_{r}\right) t}\right)\right|=O(S),
$$

(ii) $|u(x, t)-N(x, t)|<\Lambda^{-1} S t^{-1}$ for any $x$ that lies between

$$
\max \left(x_{l}(t), \lambda_{l} t-\sqrt{-2 p f^{\prime \prime}\left(u_{l}\right) t}\right) \text { and } \min \left(x_{r}(t), \lambda_{r} t+\sqrt{2 q f^{\prime \prime}\left(u_{r}\right) t}\right) \text {, }
$$

(iii) $|u(x, t)-N(x, t)|=O(S) t^{-1 / 2}$ for $x$ between $x_{l}$ and $\lambda_{l} t-\sqrt{-2 p f^{\prime \prime}\left(u_{l}\right) t}$ or between $x_{r}(t)$ and $\lambda_{r} t+\sqrt{2 q f^{\prime \prime}\left(u_{r}\right) t}$,

(iv) $u(x, t)=N(x, t)$ if $x$ lies outside the regions of (ii) and (iii), where $\lambda_{l}=f^{\prime}\left(u_{l}\right), \lambda_{r}=f^{\prime}\left(u_{r}\right)$ and $\lambda(x, t)=f^{\prime}(u(x, t))$ etc.

Proof. Given any $(x, t), x_{l}(t)<x<x_{r}(t)$ and $u$ continuous at $(x, t)$, we may construct a characteristic curve backward in time through $(x, t)$. This curve does not intersect $\chi_{1}$ or $\chi_{2}$ and thus meets $t=0$ between $x=-S / 2$ and $x=S / 2$, and

$$
x / t-f^{\prime}(u(x, t)) \leqslant S / t \text { for } x_{l}(t)<x<x_{r}(t) .
$$

It follows from this and (15) that for $(x, t)$ in the region of (ii):

$$
f^{\prime}(N(x, t))-f^{\prime}(u(x, t)) \leqslant S / t
$$

whence (ii) follows easily.

By the same arguments as in the proof of Theorem 1, the minimum of (5) must take place at $x=x^{*}(t)$ where $u\left(x^{*}(t), t\right)=u_{l}$ and thus from (16) we have

$$
\begin{gathered}
p=\min _{x} \int_{-\infty}^{x}\left(u(s, t)-u_{l}\right) d s=\int_{x_{l}(t)}^{x^{*}(t)}\left(u(s, t)-u_{l}\right) d s \\
x^{*}=t \lambda_{l}+\frac{O(S)}{t} .
\end{gathered}
$$

By Taylor expansion we have

$$
u-u_{l}=\frac{1}{f^{\prime \prime}\left(u_{l}\right)}\left[\lambda-\lambda_{l}+O\left(\lambda-\lambda_{l}\right)^{2}\right]
$$

and so it follows from (16) that

$$
\begin{aligned}
& p=\int_{x_{l}(t)}^{\lambda_{l} t+O(S) t^{-1}}\left[\frac{1}{f^{\prime \prime}\left(u_{l}\right)}\left(\frac{x}{t}-\lambda_{l}\right)+O(S) t^{-1}\right] d x \\
&=-\frac{\left(x_{l}(t)-\lambda_{l} t\right)^{2}}{2 f^{\prime \prime}\left(u_{l}\right) t}+O(S)\left(x_{l}(t)-t \lambda_{l}\right) t^{-1}+O(S) t^{-1} \\
&-2 p f^{\prime \prime}\left(u_{l}\right) t=\left(x_{l}(t)-\lambda_{l} t\right)^{2}+O(S)\left(x_{l}(t)-t \lambda_{l}\right)+O(S) t^{-1}
\end{aligned}
$$

It follows from this last estimate that $x_{l}(t)-\lambda_{l} t$ is $O\left(t^{1 / 2}\right)$, and thus if $p \neq 0$, then $\lambda_{l} t-x_{l}(t) \neq 0$ for $t$ large and 


$$
\begin{aligned}
\lambda_{l} t-x_{l}(t) & =-\sqrt{-2 p f^{\prime \prime}\left(u_{l}\right) t}+\frac{O(S)\left[x_{l}(t)-t \lambda_{l}\right]+t^{-1}}{\lambda_{l} t-x_{l}(t)+1-2 p f^{\prime \prime}\left(u_{l}\right) t} \\
& =-\sqrt{-2 p f^{\prime \prime}\left(u_{l}\right) t}+O(S) .
\end{aligned}
$$

When $p=0$, we see directly from (18) that the above estimate holds. This proves the first half of (i), the second half is proved in a similar way. Statement (iii) is an easy consequence of (i) and estimate (16). Finally, since $u(x, t)$ and $N(x, t)$ are both either $u_{l}$ or $u_{r}$ outside the regions of (ii) and (iii), (iv) follows trivially. Q.E.D.

The following corollary is a direct consequence of the above theorem.

COROLLARY. If $u_{l}=u_{r}$, then

(i)

$$
\|u(x, t)-N(x, t)\|_{L_{i}}=O(S)\left[t^{-1 / 2}+t^{-1+i / 2}\right], \quad i>0 .
$$

REMARK. We note $\|N(x, t)\|_{L_{i}}=O\left(t^{-1 / 2+1 / 2 i}\right)$ which is larger than $O\left(t^{-1 / 2}+t^{-1+i / 2}\right)$ when $t$ large. It follows that there exists no other $L_{i}$ time invariants of the solution besides $p$ and $q$. In general, when $u_{r} \geqslant u_{l}, p$ and $q$ are two $L_{\infty}$-invariants of the solution:

$$
p=\lim _{t \rightarrow+\infty}\left\{\min _{x}\left(u(x, t)-u_{l}\right)\right\}, \quad q=\lim _{t \rightarrow+\infty}\left\{\max _{x}\left(u(x, t)-u_{l}\right)\right\} .
$$

We finally remark that although we carry out our analysis for piecewise continuous solutions, by applying our techniques to approximate solutions of difference schemes we can easily prove our theorem for solutions in more general function spaces.

\section{REFERENCES}

1. C. M. Dafermos, Applications of the invariance principle for compact processes. II. Asymptotic behavior of solutions of a hyperbolic conservation law, J. Differential Equations 11 (1972), $416-424$.

2. R. J. DiPerna, Decay and asymptotic behavior of solutions to nonlinear hyperbolic systems of conservation laws, Indiana Univ. Math. J. 24 (1975), 1047-1071.

3. J. Glimm and P. D. Lax, Decay of solutions of systems of nonlinear hyperbolic conservation laws, Mem. Amer. Math. Soc., No. 101, 1970.

4. J. M. Greenberg and D. M. Tong, Decay of periodic solutions of $\partial u / \partial t+\partial f(u) / \partial x=0, J$. Math. Anal. Appl. 43 (1973), 56-71.

5. B. Keyfitz, Time-decreasing functionals of nonlinear conservation laws, Ph.D. thesis, New York Univ., 1970.

6. P. D. Lax, Hyperbolic systems of conservation laws. II, Comm. Pure Appl. Math. 10 (1957), 537-566.

7. Invariant functionals of nonlinear equations of evolution, Proc. Internat. Conf. on Functional Analysis and Related Topics (Tokyo, 1969), Univ. of Tokyo Press, Tokyo, 1970, pp. 240-251.

8. O. A. Oleinik, On uniqueness and stability of the generalized solution of the Cauchy problem for a quasilinear equation, Uspehi Math. Nauk 14 (1959), 165-170.

9. C. M. Dafermos, Characteristics in hyperbolic conservation laws. A study of the structure and the asymptotic behavior of solutions, (Lecture Notes, Heriot-Watt Univ., Edinburgh), Pitman, London (to appear).

Department of Mathematics, University of Maryland, College Park, Maryland 20742 